\title{
Doppler Assessment of Pulmonary Artery Flow Patterns and Ventricular Function After the Fontan Operation
}

\author{
Peter C. Frommelt, MD, A. Rebecca Snider, MD, Jon N. Meliones, MD, \\ and Roger $P$. Vermilion, MD
}

To assess the relation between ventricular systolic and diastolic function and pulmonary artery (PA) flow patterns after the Fontan operation, 15 postoperative patients were prospectively evaluated with echocardiography. Blood flow velocities in the PA were recorded with pulsed Doppler echocardiography. Ejection fraction was measured by 2-dimensional echocardiography using Simpson's rule. Indexes of dlastolic function were measured from the systemic atrioventricular valve inflow Doppler and included peak $E$ and $A$ velocitles, peak filling rate normallzed for stroke volume, the fractions of filling in early and late diastole (E and $A$ area fractions), and the E/A velocity and area ratios. Compared with 15 age-matched control subjects, the 15 patients who had undergone the Fontan procedure had decreased peak E velocity (0.65 \pm 0.20 vs 0.87 $\pm 0.10 \mathrm{~m} / \mathrm{s})$, decreased $E / A$ velocity ratio $(1.29$ \pm 0.23 vs $1.98 \pm 0.46)$, decreased normalized peak filling rate $(6.09 \pm 0.90$ vs $6.81 \pm 0.83$ $\left.\mathrm{s}^{-1}\right)$, decreased $E$ area fraction $(0.63 \pm 0.09$ vs $0.72 \pm 0.07)$, increased $A$ area fraction $(0.37 \pm$ 0.07 vs $0.24 \pm 0.06)$, and decreased $E / A$ area ratio (1.77 \pm 0.45 vs $3.33 \pm 1.15)(p<0.05)$. These diastolic filling abnormalities are consistent with impaired ventricular relaxation and decreased early diastolic transvalvular pressure gradient. PA Doppler recordings showed 2 distinct patterns of flow. Pattern I, observed in 9 patients, showed biphasic forward flow with peak velocitles in mid to late systole and middiastole. Pattern II, observed in the remaining 6 patients, showed decreased systolic forward flow, a late systolic to early diastolic flow reversal, and delayed onset of diastolic forward flow. Compared with pattern I patients, pattern II patients had no significant differences in any of the

From the Department of Pediatrics, C.S. Mott Children's Hospital, University of Michigan Medical Center, Ann Arbor, Michigan. Manuscript received May 20, 1991; revised manuscript received and accepted June 27, 1991.

Address for reprints: A. Rebecca Snider, MD, F 1331, C.S. Mott Children's Hospital, University of Michigan Medical Center, Ann Arbor, Michigan 48109-0204.
Doppler indexes of diastolic function; however, pattern II patients had a significantly lower ejection fraction ( $43 \pm 9$ vs $57 \pm 5 \%$ ). Thus, many patients undergoing the Fontan procedure have impaired ventricular relaxation, but, in the presence of a normal ejection fraction, biphasic forward PA flow is maintained. With the development of decreased ejection fraction, atrial systolic filling pressures are likely increased, the ventricular suction effect is decreased, and PA flow is diminished or absent in systole and early diastole.

(Am J Cardiol 1991;68:1211-1215)

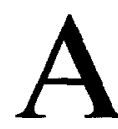
fter the Fontan operation, pulmonary artery (PA) flow is maintained in the absence of right 1 ventricular pump function. Several Doppler echocardiographic ${ }^{1-5}$ and radionuclide angiographic 6 studies have documented the contribution of right atrial contraction to pulmonary flow after direct anastomosis of the right atrium to the PA. Recently, many total cavopulmonary connections have been constructed by placement of an intraatrial conduit that excludes the right atrium from the pulmonary circuit. ${ }^{7-9}$ In these patients, postoperative invasive studies have documented adequate cardiac output and pulsatile PA flow. This suggests that changes in intrathoracic and left atrial pressure are important determinants of PA flow after the Fontan operation and that abnormalities in ventricular systolic and diastolic function can alter left atrial pressure and, thus, influence PA flow. This study assesses the relation between ventricular systolic and diastolic function and PA flow patterns after the Fontan operation using 2-dimensional and Doppler echocardiography.

\section{METHODS}

Patients: The study group included 15 patients who had undergone the Fontan operation and 15 agematched normal children. The Fontan group consisted of 11 boys and 4 girls ranging in age from 2 to 14 years (mean 5.3), in weight from 10 to $69 \mathrm{~kg}$ (mean 22.9), and in body surface area from 0.47 to $1.8 \mathrm{~m}^{2}$ (mean 
0.83 ). The control group consisted of 10 boys and 5 girls ranging in age from 4 to 15 years (mean 7.8 ), in weight from 12 to $64 \mathrm{~kg}$ (mean 28.4), and in body surface area from 0.57 to $1.8 \mathrm{~m}^{2}$ (mean 0.99 ).

The Fontan patients were examined 10 days to 8.5 years after surgery (median 2 weeks). Their diagnoses included tricuspid atresia in 4 patients, an unbalanced atrioventricular septal defect with left ventricular hypoplasia in 5 patients, double-inlet left ventricle in 2 patients, d-transposition of the great arteries with right ventricular hypoplasia in 2 patients, hypoplastic left heart syndrome after Norwood I palliation in 1 patient, and pulmonary atresia with intact ventricular septum in 1 patient.

Surgical procedure: Five patients, including all patients with tricuspid atresia and the patient with pulmonary atresia/intact ventricular septum, had direct anastomosis of the right atrial appendage to the main PA. The remaining 10 patients had a modified Fontan procedure consisting of placement of an intraatrial conduit from the inferior vena cava to the PA and direct superior vena cava to PA anastomosis. Intraoperative pressure recordings showed no significant gradient across the Fontan connection at the completion of surgery in any patient.

Echocardiographic examinations: All Fontan patients underwent a complete 2-dimensional and Doppler echocardiographic examination using a 128-element phased-array ultrasound system (Acuson, Mountain View, California) and a variety of transducers appropriate for patient size. In all the Fontan patients, Doppler color flow mapping was used to assess the amount of aortic and atrioventricular valve insufficiency. Ventricular systolic function was assessed by measuring the ejection fraction from the apical 4-chamber view using a single plane Simpson's rule algorithm. In addition, the patterns of PA blood flow velocities throughout the cardiac cycle were determined using pulsed Doppler echocardiography. PA Doppler tracings were obtained from the suprasternal, parasternal short-axis, or subcostal views, or a combination of these.

Ventricular diastolic function was evaluated in the Fontan patients and the normal children using pulsed Doppler recordings of the systemic atrioventricular valve inflow. From the apical 4-chamber view, the sample volume was positioned so as to record the maximal velocities through the valve. From the Doppler spectral recordings, the peak velocities during rapid ventricular filling (peak E) and during atrial contraction (peak A) were measured and the ratio of peak $\mathrm{E}$ to $\mathrm{A}$ velocities was calculated. To determine the pattern of ventricular filling, several areas under the Doppler spectral tracing were measured using previously described methods: (1) the total area under the velocity envelope throughout diastole, (2) the area under the Doppler curve for the first $33 \%$ of diastole $(0.33$ area ), (3) the E area or the triangular area formed by extrapolating a straight line down from the peak $E$ velocity to the baseline, and (4) the $\mathrm{A}$ area or the triangular area formed in a similar manner under the peak A velocity. ${ }^{10}$ To determine the percentage of the total velocity envelope occupied by the individual areas, the area or filling fractions were calculated as the individual areas divided by the total area under the Doppler tracing. Also, the ratio of $\mathrm{E}$ and $A$ areas was calculated. The peak filling rate normalized for stroke volume was calculated as the peak $\mathrm{E}$ velocity divided by the total velocity time integral. ${ }^{11}$

All Doppler examinations were recorded at a paper speed of $100 \mathrm{~mm} / \mathrm{s}$. The Doppler areas were traced from the paper recording using a digitizing tablet with a crosswire cursor, a personal computer, and commercially available computer software (Freeland Medical Systems, Boulder, Colorado). The Doppler velocities and areas were measured by tracing the outermost border of the spectral recordings.

Statistical analysis: Three cardiac cycles were measured and averaged to obtain each atrioventricular valve Doppler index and to calculate the ejection fraction. Statistical comparisons of the systemic atrioventricular valve inflow Doppler measurements between the Fontan patients and the normal children were made using paired $t$ tests and Bonferroni's correction for multiple comparisons. The Fontan patients were divided into 2 subgroups based on the pattern of PA blood flow, and comparisons of ejection fraction and systemic atrioventricular valve Doppler indexes were obtained between the 2 subgroups using unpaired $t$ tests. A 2tailed $p$ value $<0.05$ was used to indicate a significant intergroup difference. All values are mean \pm standard deviation.

\section{RESULTS}

Patients: The Fontan patients did not differ from the normal children in age, weight, or body surface area; however, their heart rates were significantly higher $(97 \pm 19$ vs $81 \pm 13$ beals $/ \mathrm{min}, \mathrm{p}=0.01)$. Of the 15 Fontan patients, 12 had no aortic insufficiency, 1 had trivial to mild aortic insufficiency, and 2 had moderate to severe aortic insufficiency. One of these latter 2 patients also had moderate to severe atrioventricular valve insufficiency. The remaining 14 patients had trivial to mild $(n=11)$ or no $(n=3)$ atrioventricular valve insufficiency. Ventricular systolic function was mildly depressed with an ejection fraction of $51 \pm 6.9 \%$ (range 37 to $64 \%$ ) in the Fontan group.

Diastolic function in the Fontan patients: Compared with normal children, the Fontan patients had abnormal atrioventricular valve Doppler recordings (Table I). 
In the Fontan patients, the peak $\mathrm{E}$ velocity and $\mathrm{E}$ area fraction were significantly decreased, and the $\mathrm{A}$ area fraction was significantly increased. This resulted in a decrease in the E/A velocity and area ratios. In addition, the Fontan patients had a decreased normalized peak filling rate.

Pulmonary artery flow patterns in the Fontan patients: PA Doppler recordings revealed 2 distinct patterns of flow in the Fontan patients. Pattern I, observed in 9 patients, was characterized by biphasic forward PA flow with peak velocities in mid to late systole and mid-diastole (Figure 1). Nadirs in pulmonary flow occurred 50 to $100 \mathrm{~ms}$ after mitral valve opening and 50 to $100 \mathrm{~ms}$ after atrial contraction. Pattern II, observed in the remaining 6 patients, was characterized by di-

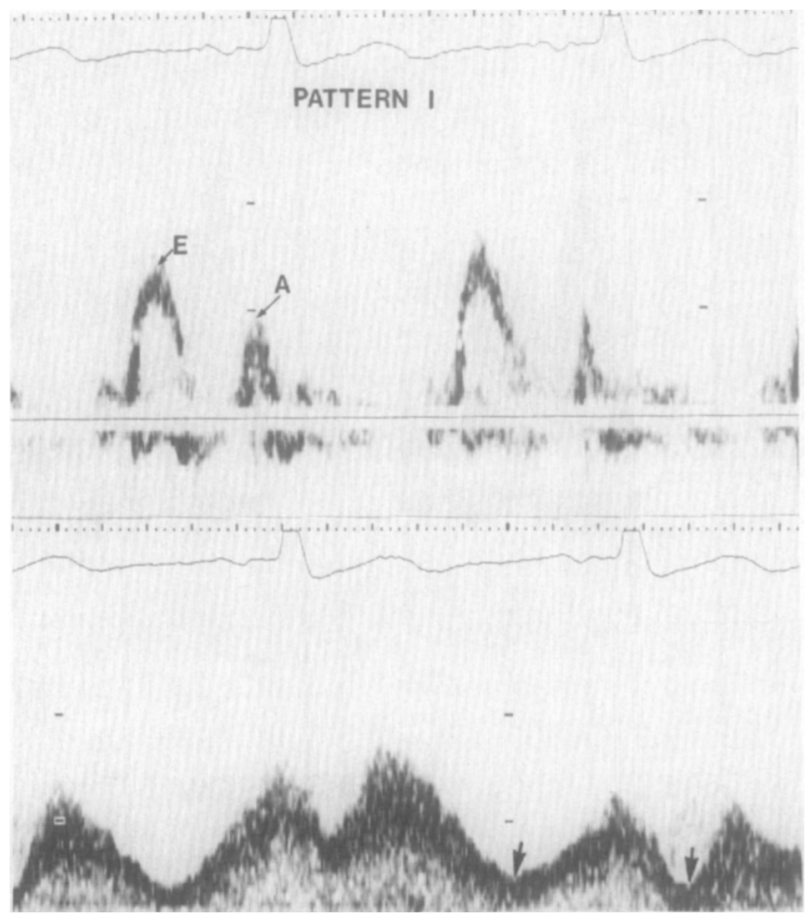

FIGURE 1. Atrioventricular valve Doppler recording (top) and pulmonary artery (PA) Doppler recording (bottom) from a pafient with pattem I PA flow after a modified Fontan procedure (intraatrial conduit). The atrioventricular valve Doppler recording was obtained nearly simultaneously with the PA Doppler recording for purposes of timing of fiow patterns. On the PA Doppler tracing, systolic flow is initiated with left atrial relaxation and is accentuated during midsystole by a ventricular suction effect as the atrioventricular valve is pulled downward toward the ventricular apex. As the left atrial pressure increases in late systole, the transpulmonary gradient decreases, leading to a decrease in forward pulmonary blood flow. Forward flow again increases in mid-diastole as left atrial pressure decreases with atrioventricular valve opening. With atrial contraction in late diastole, left atrial pressure increases rapidly, resulting in decreases in transpulmonary gradient and forward pulmonary flow. Nadirs in pulmonary flow (arrows) occur at 50 to $\mathbf{1 0 0} \mathrm{ms}$ after atrioventricular valve opening and 50 to 100 ms after atrial contraction. These minimal flow velocities in the PA occur at times when left atrial pressure is highest, at the end of systole before atrioventricular valve opening and with left atrial contraction. $A=$ peak velocity during atrial contraction; $E$ = peak velocity during rapid ventricular filling.

\begin{tabular}{|c|c|c|c|}
\hline & Normal Subjects & Fontan Patients & $p$ Value \\
\hline Peak E (nis) & $0.87 \pm 0.10$ & $0.65 \perp 0.20$ & $0.0006^{\star}$ \\
\hline Peak A $(\mathrm{m} / \mathrm{s})$ & $0.46 \pm 0.09$ & $0.51 \pm 0.12$ & 0.25 \\
\hline Peak E/peak A & $1.98 \pm 0.46$ & $1.29 \pm 0.23$ & $0.0001^{*}$ \\
\hline 0.33 area $(\mathrm{m})$ & $0.072 \pm 0.015$ & $0.049 \pm 0.02$ & $0.001^{*}$ \\
\hline E area $(m)$ & $0.091 \pm 0.02$ & $0.067 \pm 0.02$ & $0.002^{*}$ \\
\hline A area $(m)$ & $0.031 \pm 0.01$ & $0.039 \pm 0.01$ & 0.095 \\
\hline E area fx & $0.72 \pm 0.07$ & $0.53 \pm 0.09$ & $0.01^{*}$ \\
\hline A area $f x$ & $0.24 \pm 0.06$ & $0.37 \pm 0.07$ & $0.0001^{*}$ \\
\hline E area/A area & $3.33 \pm 1.15$ & $1.77 \pm 0.45$ & $0.0001^{*}$ \\
\hline PFR/SV (SV/S) & $6.81 \pm 0.83$ & $6.09 \pm 0.90$ & $0.03^{\star}$ \\
\hline
\end{tabular}

minished forward systolic pulmonary flow, reversed late systolic to early diastolic flow, and delayed onset of forward diastolic flow (Figure 2). Forward PA flow was augmented during inspiration in all Fontan patients, but the pattern of flow was not influenced by respiration. The 5 patients with direct right atrial to PA connections had pattern I PA flow. Four of the 10 patients with an intraatrial cavopulmonary connection also had pattern I PA flow; the other 6 patients had pattern II PA flow.

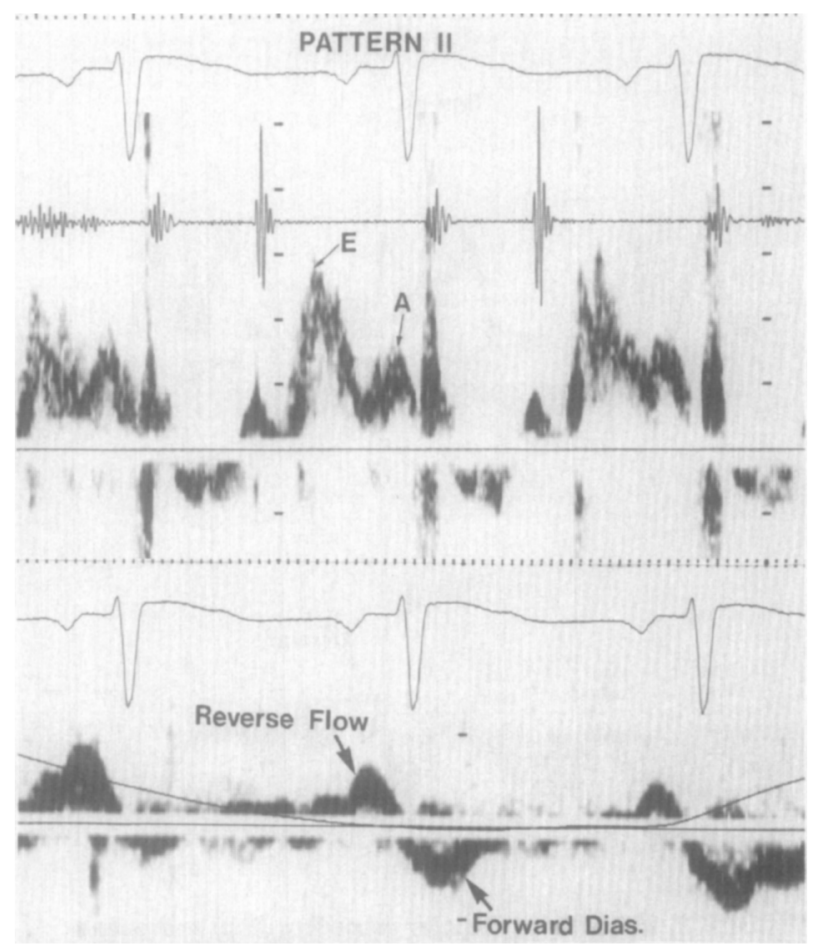

FIGURE 2. Atrioventricular valve Doppler recording (top) and pulmonary artery (PA) Doppler recording (bottom) from a patient with pattern II PA fiow after Fontan operation. The inflow Doppler recording was obtained nearly simultaneously with the PA Doppler recording in order to determine timing of flow in the PA. Pattern II flow is characterized by diminished forward systolic pulmonary flow, reversed late systolic to earIy diastolic (Dias.) flow, and delayed onset of forward diastolic flow. Abbreviations as in Figure 1. 
Subtle variations in the 2 PA flow patterns were identified. Among the patients with pattern I PA flow, patients with a direct right atrial to PA connection had accentuated late diastolic forward flow secondary to right atrial contraction and a short interval of reversed flow in early systole secondary to right atrial relaxation (Figure 3). These subtle deflections were not observed in pattern I patients with an intraatrial cavopulmonary connection. The 1 patient with severe aortic and atrioventricular valve insufficiency had pattern II PA flow with diminished systolic forward flow and reversed late systolic to early diastolic flow; however, this patient also had reversed flow in late diastole to early systole (Figure 4).

Compared with pattern I patients, pattern II patients had no significant differences in any of the Doppler indexes of diastolic function; however, pattern II patients had a significantly lower ejection fraction ( $43 \pm$ 9 vs $57 \pm 5 \%$ in the pattern I group, p <0.003). Three of the 6 patients with pattern II PA flow have had a poor outcome after the Fontan operation. The patient with severe aortic and atrioventricular valve insufficiency required atrioventricular valvuloplasty and aortic valve replacement. Two patients had chronic low cardiac output postoperatively - 1 subsequently underwent

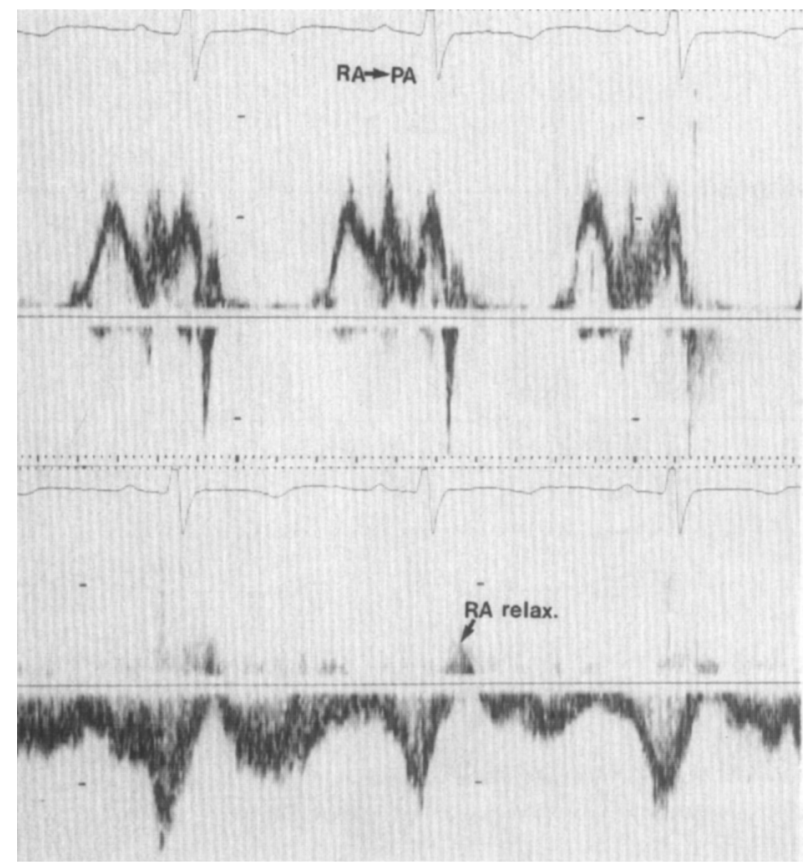

FIGURE 3. Mitral valve Doppler recording (top) and pulmonary artery (PA) Doppler recording (bottom) from a patient with pattem I PA flow after Fontan operation with direct right atrial (RA) to PA connection. In this echocardiogram, forward flow in the PA is shown below the baseline and reverse flow in the PA is shown above the baseline. As in Figure 1, there is biphasic forward flow with peak velocities in mid-10-late systole and mid-diastole. Pattem I patients with RA to PA connection differed slightly from pattern I patients with an intraatrial conduit. These patients had accentuated late diastolic forward flow secondary to RA contraction and a short interval of reverse flow in early systole secondary to RA relaxation. cardiac transplantation and the other continues to have diminished exercise tolerance and clinical evidence of systemic venous hypertension. All patients with pattern I PA flow are asymptomatic and have required no reoperations.

\section{DISCUSSION}

In this study, we examined the relation between ventricular systolic and diastolic function and PA blood flow velocities after the Fontan operation. Compared with normal children, Fontan patients have decreased early diastolic ventricular filling with a compensatory increase in filling at atrial contraction consistent with impaired ventricular relaxation and a decreased early
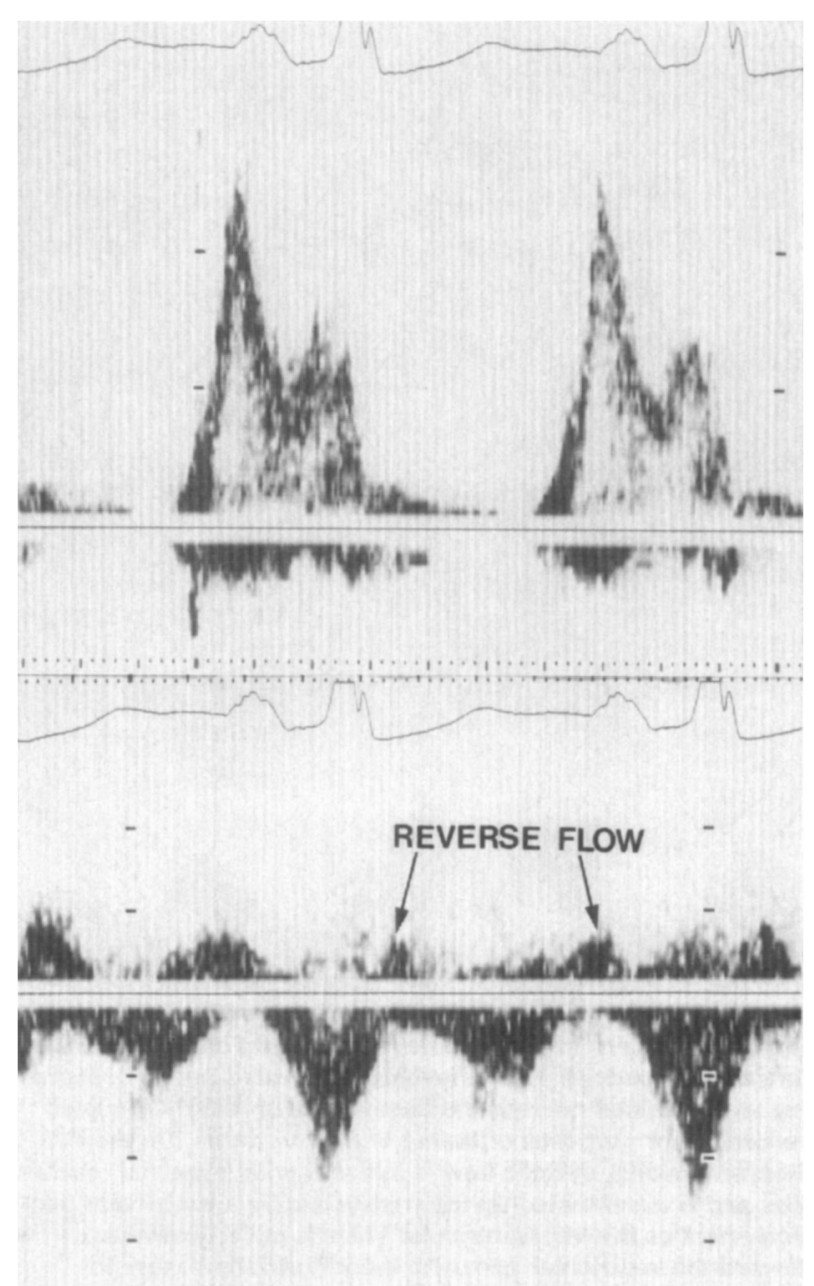

FIGURE 4. Infiow Doppler recording (top) and pulmonary artery (PA) Doppler recording (bottom) from a patient with severe aortic and atrioventricular valve insufficiency after Fontan operation. The inflow Doppler recording was obtained nearty simultaneously with the PA Doppler recording for purposes of timing of flow. In this example, forward fiow in the PA is shown below the baseline. This patient demonstrated pattem II PA flow with diminished systolic forward flow and reversed late systolic to early diastolic flow. However, this patient also had reverse flow in late diastole to early systole. These $\mathbf{2}$ flow reversals are probably related to severe aortic and atrioventricular valve insufficiency with resultant alterations in left ventricular diastolic and left atrial systolic pressures. 
diastolic transvalvular pressure gradient. Diastolic filling abnormalities were found in all Fontan patients; however, diastolic filling indexes did not distinguish the 2 different patterns of PA blood flow velocities found in the Fontan patients. These patterns appear to be strongly influenced by ventricular systolic function, with diminished forward systolic pulmonary flow and delayed onset of forward diastolic flow in patients with a decreased ejection fraction (pattern II). With wellpreserved systolic function, biphasic forward pulmonary flow is maintained (pattern I).

Possible mechanisms of diastolic filling abnormalitles: Several studies have described increased ventricular dimensions and diminished contractility before and after the Fontan operation in patients with a functional single ventricle. ${ }^{12-14}$ With dilatation and decreased contractility of the ventricle, systolic filling of the atrium is decreased secondary to diminished downward displacement of the atrioventricular valve during ventricular contraction. This results in reduced early diastolic atrial preload, a decreased early diastolic transvalvular pressure gradient and decreased early ventricular filling. Increased end-systolic ventricular volume also results in decreased early diastolic flow and a shift in filling to late diastole caused by diminished ventricular relaxation and elevated early diastolic ventricular filling pressures.

Factors influencing pulmonary artery flow patterns: The biphasic forward flow found in pattern I patients correlated well with the biphasic changes in left atrial pressure that occur during the cardiac cycle (Figure 1). Systolic PA flow is initiated in early systole at the time of left atrial relaxation and is accentuated during midsystole, presumably by the ventricular suction effect as the atrioventricular valve is pulled downward toward the ventricular apex. As left atrial pressure increases in late systole, the transpulmonary gradient decreases with a resultant decrease in forward PA flow. PA flow again increases in mid-diastole as left atrial pressure decreases with atrioventricular valve opening. At atrial contraction in late diastole, left atrial pressure increases abruptly, resulting in a decrease in the transpulmonary gradient and forward PA flow. Nadirs in PA flow occurred 50 to $100 \mathrm{~ms}$ after atrioventricular valve opening and 50 to $100 \mathrm{~ms}$ after atrial contraction. These minimal flow velocities in the $P \Lambda$ occur at times of highest left atrial pressure (at the end of systole before atrioventricular valve opening and with atrial contraction). The timing of these events lend support to the hypothesis that variations in PA flow are related to changes in left atrial pressure with a short delay as these pressure changes are transmitted across the pulmonary bed.

Pattern II patients had diminished systolic PA flow and delayed onset of forward diastolic flow in associa- tion with decreased systolic ventricular function. These findings suggest that with diminished ventricular systolic function, left atrial pressure was increased and the transpulmonary gradient was decreased secondary to poor ventricular emptying and diminished atrioventricular valve excursion during systole. As a result, forward PA flow was decreased during systole and early diastole.

Clinical implications of pulmonary artery Doppler patterns: Identification of PA flow patterns after the Fontan operation in conjunction with an assessment of ventricular function can be a useful predictor of clinical outcome. ${ }^{1}$ Three of the 6 patients with pattern II PA flow had a poor outcome after Fontan. All the patients with pattern I flow are doing well clinically.

Acknowledgment: We thank Kathlene Chmielewski, CMA, for editorial assistance, and Jane Peters and Lyne Merida for technical assistance in the preparation of this manuscript.

\section{REFERENCES}

1. Hagler DJ, Seward JB, Tajik AJ, Ritter DG. Functional assessment of the Fontan operation: combined M-mode, two-dimensional, and Doppler echocardiographic studics. J Am Coll Cardiol 1984;4:756-764.

2. Nakazawa M, Nakanishi T, Okuda H, Satomi G, Nakae S, Imai Y, Takao A. Dynamics of right heart flow in patients after Fontan procedure. Circulation 1984;69:306-312.

3. DiSessi TG, Child JS, Perloff JK, Wu L, Williams RG, Laks H, Friedman WF. Systemic venous and pulmonary arterial flow patterns after Fontan's procedure for tricuspid atresia or single ventricle. Circulation 1984;70:898-902.

4. Nakazawa M, Nojima K, Okuda H, Imai Y, Nakanishi T, Kurosawa H, Takao A. Flow dynamics in the main pulmonary artery after the Fontan procedure in patients with tricuspid atresia or single ventricle. Circulation 1987;75:1117-1123. 5. Queshi SA, Richheimer R, McKay R, Arnold R. Doppler echocardiographic evaluation of pulmonary artery flow after the Fontan operation: importance of atrial contraction. Br Heart $J$ 1990;64:272-276.

6. Jamos GG, Gelfand MJ, Schwartz DC, Kaplan S. Postoperative evaluation of the Fontan procedure by radionuclide angiography. Am Heart $J$ 1982;104: 785-793.

7. Puga FJ, Chiavarelli M, Hagler DJ. Modifications of the Fontan operation applicable to patients with left atrioventricular valve atresia or single atrioventricular valve. Circulation 1987;76(suppl III):III-53-III-60.

8. de Leval MR, Kilner P, Gewillig M, Bull C, McGoon DC. Total cavopulmonary connection: a logical alternative to atriopulmonary connection for complex Fontan operations. J Thorac Cardiovasc Surg 1988;96:682-695.

9. Jonas RA, Castaneda AR. Modified Fontan procedure: atrial baffle and systemic venous to pulmonary artery anastomotic techniques. I Cardiac Surg 1988:3:91-96.

10. Snider AR, Gidding SS, Rocchini AP, Rosenthal A, Dick M, Crowley DC, Peters J. Doppler evaluation of left ventricular diastolic filling in children with systemic hypertension. Am J Cardiol 1985;56:921-926.

11. Bowman LK, Forrester AL, Jaffe CC, Mattera J, Wackers FJT, Zaret BL. Peak filling normalized to mitral stroke volume: a new Doppler echocardiographic filling index validated by radionuclide angiographic techniques. $\mathrm{J} \mathrm{Am} \mathrm{Coll} \mathrm{Car-}$ diol 1988;12:937-943.

12. Gewillig MH, Lundstrom UR, Deanfield JE, Bull C, Franklin RC, Graham TP, Wyse RK. Impact of Fontan operation on left ventricular size and contractility in tricuspid atresia. Circulation 1990;81:118-127.

13. Nakae S, Imai $Y$, Harada $Y$, Sawatari $K$, Kawada M, Takanashi $Y$, Ishihara K, Hashimoto A, Hayashi H, Koyanagi H, Kanaya M, Nakazawa M, Takao A. Assessment of left ventricular function before and after Fontan's operation for the correction of tricuspid atresia. Heart Vessels 1985;1:83-88.

14. La Corte MA, Dick M II, Scheer G, La Farge CG, Fyler DC. Left ventricular function in tricuspid atresia. Circulation 1975;52:996-1000. 